\title{
Inferences for Weibull lifetime model under progressively first-failure censored data with binomial random removals
}

\author{
Samir K. Ashour ${ }^{1}$, Ahmed A. El-Sheikh ${ }^{1}$, Ahmed Elshahhat ${ }^{2, *}$ \\ ${ }^{1}$ Faculty of Graduate Studies for Statistical Research, Cairo University, Egypt \\ ${ }^{2}$ Faculty of Technology and Development, Zagazig University, Egypt
}

\begin{abstract}
In this paper, the Bayesian and non-Bayesian estimations of a two-parameter Weibull lifetime model in presence of progressive first-failure censored data with binomial random removals are considered. Based on the s-normal approximation to the asymptotic distribution of maximum likelihood estimators, two-sided approximate confidence intervals for the unknown parameters are constructed. Using gamma conjugate priors, several Bayes estimates and associated credible intervals are obtained relative to the squared error loss function. Proposed estimators cannot be expressed in closed forms and can be evaluated numerically by some suitable iterative procedures. A Bayesian approach is developed using Markov chain Monte Carlo techniques to generate samples from the posterior distributions and in turn computing the Bayes estimates and associated credible intervals. To analyze the performance of the proposed estimators, a Monte Carlo simulation study is conducted. Finally, a real data set is discussed for illustration purposes.
\end{abstract}

Keywords Bayes Procedure, Markov Chain Monte Carlo, Maximum Likelihood Estimation, Progressive First-Failure Censored Sampling, Squared Error Loss Function, Weibull Distribution

AMS 2010 subject classifications 62F10, 62F15, 62N01, 62N02, 62N05

DOI: $10.19139 /$ soic-2310-5070-611

\section{Introduction}

In life-testing experiments and reliability studies, experimenters wish to observe the failure times of units placed on test. But due to time or cost or due to some other unavoidable circumstances, experimenters terminate a study before recording the responses of all the units under study. This results to availability of censored data. Type-I (time censoring) and Type-II (failure censoring) are the most common and popular censoring schemes in life-testing experiments. A generalization of Type-II censoring is Type-II progressive censoring scheme (PCS). However, when the lifetime of an item is very high and test facilities are limited but test material is relatively less expensive, the experimenter can be test $n$ sets with $k$ items within each set. This life-test is then conducted by testing each of these sets of units separately until the occurrence of first-failure in each set. Such a censoring scheme is called first-failure censoring, see [7]. Under this scheme, one can save a considerable amount of time as well as money. Unfortunately, the first-failure censoring is do not allow for sets to be removed from the test at points other than the final termination point. Intermediate removal may be desirable when a compromise between the reduced time of experimentation and the observation of at least some extreme lifetimes is sought or when some of the surviving units in the experiment that are removed early on can be used for some other tests, see [5]. For this reason, [26] proposed a life-test plan called progressively first-failure censored sampling (PFFCS) which is a combination between the concepts of first-failure censoring and Type-II PCS. This censoring plan is modifies

\footnotetext{
*Correspondence to: Ahmed Elshahhat (Email: dr_ahmed_elshahhat@yahoo.com; a.elshahat@zu.edu.eg). Department of Accounting and Quantitative Information Systems, Zagazig University, Zagazig, Sharkia, Egypt (44519).
}

ISSN 2310-5070 (online) ISSN 2311-004X (print)

Copyright (C) 2021 International Academic Press 
first-failure censoring by allowing the experimenter to remove a pre-specified number of surviving groups from a life-test. Also, they discussed the maximum likelihood estimators (MLEs) and expected test time for the unknown parameters of Weibull distribution (WD) based on the PFFCS.

Recently, the PFFCS has become quite popular in a life-testing problem and reliability analysis. However, the estimation of parameters of different lifetime models based on PFFCS have been studied by several authors such as $[11,20,2,4,1]$. In some practical situations, the progressive censoring schemes with random removals are needed. For example, consider a clinical test that a doctor perform an experiment with $n$ cancer patients but after the death of first patient, some patient leave the experiment and go for treatment to other doctor/hospital. Similarly, after the second death a few more leave and so on. Finally the doctor stops taking observations as soon as the predetermined number of deaths (say $m$ ) are recorded. Hence, the number of patients drop out from a hospital at each stage is random and cannot be pre-determined. It may be assumed that in each stage, the participating patients may independently decide to leaving the experiment with the same probability $(p)$ for all the patients. Hence, the number of patients who leave the experiment at a specified stage will follow the binomial distribution with probability of success $(p)$. For this reason, $[15,23]$ have considered the problem of estimation when the number of units removed at each stage follows a discrete uniform and binomial distributions, respectively, for progressively first-failure censored data.

It should be noted that the discrete uniform distribution does not increase the difficulty in estimating the model parameters as compared to the cases with a fixed removal pattern, becuase it does not introduce any additional parameter to the likelihood function. Nevertheless, a discrete uniform removal pattern may not seem very realistic as it assumes that each removal event occurs with an equal probability regardless of the number of units removed. A more realistic alternative to describe the number of occurrences of an event out of $n$ trials is the binomial distribution as suggested by [24]. [15] discussed the estimation problems of Pareto distribution when the lifetimes data are collected under a PFFCS with uniform removals. [23] derived the MLEs and Bayes estimators (BEs) of a parameter of Burr-X model under progressively first-failure censoring scheme with binomial removals (PFFCS$\mathrm{BR}$ ), were the number of units removed at each failure time has a discrete binomial distribution with certain probability $p$. Many several authors considered the problem of statistical inference based on Type-II progressive censoring with binomial random removals, see [24, 25, 10]. Recently, [8] discussed the Bayesian analysis of competing risk data under Type-II PCS where the number of units removed at each stage has a binomial distribution. For further details, an excellent book dedicate to the PCSs was proposed by [5], can be recommended to the readers.

[26] proposed PFFCS as a generalization of Type-II PCS. Suppose that $n$ independent groups with $k$ items within each group are put on a life-testing experiment at time zero, $m$ is a pre-fixed number of failures, and the PCS $\mathbf{R}=\left(R_{1}, R_{2}, \ldots, R_{m}\right)$, is pre-fixed. At the time of first observed failure (say $\left.X_{1}\right), R_{1}$ groups and the group in which the first failure are randomly removed from the experiment. Following the second observed failure (say $\left.X_{2}\right), R_{2}$ groups and the group in which the second failure is observed are randomly removed from the remaining live $n-R_{1}-1$ groups, and so on. This procedure continues until all remaining live $R_{m}$ groups and the group in which the $m-t h$ failure are removed at the time of the $m-t h$ failure has occurred. Suppose that the failure times of $n \times k$ items originally in the test are from a continuous population with cumulative distribution function (CDF), $F(x ; \theta)$, and probability density function (PDF), $f(x ; \theta)$. Let $\left(X_{(i)}, R_{i}\right), i=1,2, \ldots, m$ denote a progressively first-failure censored sample, where $X_{(1)}<X_{(2)}<\cdots<X_{(m)}$ with pre-determined number of removals, say $R_{1}=r_{1}, R_{2}=r_{2}, \ldots, R_{m}=r_{m}$. Then, the conditional likelihood function of PFFCS can be written as (see [26])

$$
L_{1}(\theta \mid x, \mathbf{R}=r)=C k^{m} \prod_{i=1}^{m} f\left(x_{(i)} ; \theta\right)\left[1-F\left(x_{(i)} ; \theta\right)\right]^{k\left(r_{i}+1\right)-1},
$$

where $C=n\left(n-r_{1}-1\right) \ldots\left(n-\sum_{i=1}^{m}\left(r_{i}+1\right)\right)$ and $n=m+\sum_{i=1}^{m} r_{i}$. From (1), some sampling schemes can be obtained as a special cases, such as: Type-II PCS by putting $k=1$, the joint PDF of the first-failure censoring by putting $\mathbf{R}=(0,0, \ldots, 0)$, Type-II censoring by putting $\mathbf{R}=(0,0, \ldots, n-m)$ and $k=1$, and if putting $\mathbf{R}=(0,0, \ldots, 0)$ and $k=1$, then $n=m$, which yields the complete sampling.

Now, suppose that an individual group being removed from the progressively first-failure censored life-test is independent of the others but with the same probability $p$. Then, the number $r_{i}$ of groups removed at the $i-t h$ failure for $i=1,2, \ldots, m-1$, where $m$ is predetermined before the life-testing, follows a binomial distribution 
with parameters $n-m-\sum_{j=1}^{i-1} r_{j}$ and $p$, see [23]. Therefore,

$$
P\left(R_{1}=r_{1}\right)=\left(\begin{array}{c}
n-m \\
r_{1}
\end{array}\right) p^{r_{1}}(1-p)^{n-m-r_{1}},
$$

and for $i=2,3, \ldots, m-1$,

$$
P\left(R_{i}=r_{i} \mid R_{i-1}=r_{i-1}, \ldots, R_{1}=r_{1}\right)=\left(\begin{array}{c}
n-m-\sum_{j=1}^{i-1} r_{j} \\
r_{i}
\end{array}\right) p^{r_{i}}(1-p)^{n-m-\sum_{j=1}^{i-1} r_{j}},
$$

where $0 \leqslant r_{1} \leqslant n-m$ and $0 \leqslant r_{i} \leqslant n-m-\sum_{j=1}^{i-1} r_{j}$ for $i=2,3, \ldots, m-1$.

Furthermore, we assume that $\mathbf{R}=\left(R_{1}, R_{2}, \ldots, R_{m}\right)$ is independent of $X_{(i)}$ for all $i=1,2, \ldots, m$. Hence the likelihood function takes the following form

$$
L(\theta, p \mid x, r)=L_{1}(\theta \mid x, \mathbf{R}=r) L_{2}(\mathbf{R}=r ; p),
$$

where $L_{2}(\mathbf{R} ; p)$ is the joint probability distribution of binomial removals, is given by

$$
\begin{aligned}
L_{2}(\mathbf{R}=r ; p) & =P\left(R_{1}=r_{1}\right) P\left(R_{2}=r_{2} \mid R_{1}=r_{1}\right) P\left(R_{3}=r_{3} \mid R_{2}=r_{2}, R_{1}=r_{1}\right) \\
& \times \cdots \times P\left(R_{m-1}=r_{m-1} \mid R_{m-2}=r_{m-2}, \ldots, R_{1}=r_{1}\right) .
\end{aligned}
$$

Substituting (2) and (3) into (5), we get

$$
L_{2}(\mathbf{R}=r ; p)=\frac{(n-m) !}{\left(n-m-\sum_{i=1}^{m-1} r_{i}\right) ! \prod_{i=1}^{m-1} r_{i} !} p^{\sum_{i=1}^{m-1} r_{i}}(1-p)^{(m-1)(n-m)-\sum_{i=1}^{m-1}(m-i) r_{i}} .
$$

From (4), it can be seen that $L_{1}$ does not involved the binomial parameter $p$ and can be treated as function of $\theta$ only, therefore, $L_{1}$ can be maximizing directly by obtained the MLE of $\theta$. On the other hand, $L_{2}$ involves $p$ only, hence, the MLE $\hat{p}$ of $p$ can be obtained by maximizing $L_{2}$ directly. The sampling procedure for a life-test based on PFFCS-BR, is illustrated in Table 1.

Table 1. Sampling procedure for a life-test under PFFCS-BR.

\begin{tabular}{cccc}
\hline Stage & Failure item & Removed group(s) & Survived group(s) \\
\hline 1 & $X_{(1)}$ & $r_{1} \sim \operatorname{Bin}(n-m, p)$ & $n-r_{1}-1$ \\
2 & $X_{(2)}$ & $r_{2} \sim \operatorname{Bin}\left(n-m-r_{1}, p\right)$ & $n-r_{1}-r_{2}-2$ \\
$\vdots$ & $\vdots$ & $\vdots$ & $\vdots$ \\
$m-1$ & $X_{(m-1)}$ & $r_{m-1} \sim \operatorname{Bin}\left(n-m-\sum_{j=1}^{m-2} r_{j}, p\right)$ & $n-(m-1)-\sum_{j=1}^{m-1} r_{j}$ \\
$m$ & $X_{(m)}$ & $r_{m}=n-m-\sum_{j=1}^{m-1} r_{j}$ & 0 \\
\hline
\end{tabular}

The WD is one of the most widely used distributions in the reliability and survival studies due to its various shapes of the hazard function. This model has been extensively used to model lifetimes and material strengths, see [16]. Suppose that the observed failure times are independent identically distributed with $\operatorname{WD}(\alpha, \lambda)$, where $\alpha$ and $\lambda$ are the shape and scale parameters, respectively. Then, the PDF and CDF of WD, are given, respectively, by

$$
f(x ; \alpha, \lambda)=\alpha \lambda x^{\alpha-1} e^{-\lambda x^{\alpha}}, \quad x>0, \alpha, \lambda>0,
$$

and

$$
F(x ; \alpha, \lambda)=1-e^{-\lambda x^{\alpha}}, \quad x>0, \alpha, \lambda>0 .
$$


In this paper, our main purpose is to use the maximum likelihood method and Bayes procedure to derive both point and interval estimates of the unknown parameters of WD under PFFCS-BR. Based on the squared error loss (SEL) function, the BEs are developed. When both parameters are unknown, the MLEs and BEs cannot be obtained in explicit forms, as expected, but can be evaluated numerically. Also, approximate confidence intervals (ACIs) and Bayes credible intervals (BCIs) for the unknown parameters are constructed. Based on Markov chain Monte Carlo (MCMC) techniques, namely Gibbs sampler and Metropolis-Hastings (M-H) algorithm, the Bayes estimates of the unknown parameters and associated BCIs are obtained. Monte Carlo simulation study is performed to compare the performances among various estimates in terms of their mean square error (MSE) and average confidence lengths (ALs). Furthermore, a numerical example with real data set is discussed to illustrate our proposed methods.

The rest of the paper is organized as follows: In Sections 2 and 3, the MLEs and BEs of the unknown parameters are investigated, respectively. In Section 4 we provide a simulation study. A real life-data set has been analyzed in Section 5. Finally, the concluding remarks and different special cases obtained from the new results are presented in Section 6.

\section{Maximum Likelihood Estimation}

This section discusses the procedures of obtaining the MLEs of the parameters $\alpha, \lambda$ and $p$, as well as corresponding $100(1-\gamma) \%$ two-sided ACIs, based on PFFCS-BR. Suppose that $n \times k$ independent units taken from a population are placed on a PFFCS-BR life-test with the corresponding lifetimes being identically distributed having PDF and $\mathrm{CDF}$ as defined in (7) and (8), respectively. Substituting from (7) and (8) into (1), the likelihood function (1) can be written with proportional as

$$
L_{1}(\alpha, \lambda \mid x, r) \propto(\alpha \lambda)^{m} \exp \left(-\lambda k \sum_{i=1}^{m}\left(r_{i}+1\right) x_{(i)}^{\alpha}\right) \prod_{i=1}^{m} x_{(i)}^{\alpha-1},
$$

where $C$ is defined in (1). Taken the natural logarithm of (9), $\ell_{1}(\cdot)=\log L_{1}(\cdot)$, we get

$$
\ell_{1}(\alpha, \lambda \mid x, r) \propto m \log (\alpha \lambda)-\lambda k \sum_{i=1}^{m}\left(r_{i}+1\right) x_{(i)}^{\alpha}+(\alpha-1) \sum_{i=1}^{m} \log x_{(i)} .
$$

Differentiating (10) with respect to $\alpha$ and $\lambda$, and equating each result to zero, two normal equations must be solving simultaneously to obtain the MLEs $\hat{\alpha}$ and $\hat{\lambda}$ of $\alpha$ and $\lambda$, respectively.

Similarly, since (6) does not involve the parameters $\alpha$ and $\lambda$, the MLE $\hat{p}$ of $p$ can be found by maximizing (6) directly. Hence, the natural logarithm of $(6), \ell_{2}(\cdot)=\log L_{2}(\cdot)$ can be written up to proportional as

$$
\ell_{2}(\mathbf{R}=r ; p) \propto \sum_{i=1}^{m-1} r_{i} \log (p)+\left((m-1)(n-m)-\sum_{i=1}^{m-1}(m-i) r_{i}\right) \log (1-p) .
$$

The first order derivative of (11) with respect to $p$ will be

$$
\frac{\partial \ell_{2}}{\partial p}=\frac{\sum_{i=1}^{m-1} r_{i}}{p}-\frac{\left((m-1)(n-m)-\sum_{i=1}^{m-1}(m-i) r_{i}\right)}{(1-p)} .
$$

Using (12), independently, the MLE $\hat{p}$ of $p$ becomes

$$
\hat{p}=\frac{\sum_{i=1}^{m-1} r_{i}}{\left((m-1)(n-m)-\sum_{i=1}^{m-1}(m-i-1) r_{i}\right)} .
$$

Clearly, the MLEs $\hat{\alpha}$ and $\hat{\lambda}$ cannot be solved analytically but can be evaluated numerically, therefore numerical methods like the Newton-Raphson method are required to solve $\hat{\alpha}$ and $\hat{\lambda}$ iteratively. 


\subsection{Approximate Interval Estimation}

The $100(1-\gamma) \%$ two-sided ACIs based on asymptotic distributions of the MLEs $\hat{\alpha}, \hat{\lambda}$ and $\hat{p}$ of the parameters $\alpha$, $\lambda$ and $p$, respectively, are derived. The asymptotic variances and covariances of the MLEs are given by elements of the inverse of Fisher information matrix. The Fisher information matrix, $\mathrm{I}(\underline{\theta})$, associated with $\alpha, \lambda$ and $p$ is given by

$$
\mathrm{I}(\underline{\theta})=\left[\begin{array}{cc}
\mathrm{I}_{1}(\alpha, \lambda) & 0 \\
0 & \mathrm{I}_{2}(p)
\end{array}\right]=E\left[\begin{array}{ccc}
-\frac{\partial^{2} \ell_{1}}{\partial \alpha} & -\frac{\partial^{2} \ell_{1}}{\partial \alpha \partial \lambda} & 0 \\
-\frac{\partial^{2} \ell_{1}}{\partial \lambda \partial \alpha} & -\frac{\partial^{2} \ell_{1}}{\partial \lambda^{2}} & 0 \\
0 & 0 & -\frac{\partial^{2} \ell_{2}}{\partial p^{2}}
\end{array}\right]
$$

where $\underline{\theta}=(\alpha, \lambda, p)^{\mathbf{T}}$. Hence, from (10) and (11), the elements of (13) becomes

$$
\begin{aligned}
& \frac{\partial^{2} \ell_{1}}{\partial \alpha^{2}}=-\frac{m}{\alpha^{2}}-\lambda k \sum_{i=1}^{m}\left(r_{i}+1\right) x_{(i)}^{\alpha}\left(\log x_{(i)}\right)^{2}, \frac{\partial^{2} \ell_{1}}{\partial \lambda^{2}}=-\frac{m}{\lambda^{2}}, \\
& \frac{\partial^{2} \ell_{1}}{\partial \alpha \partial \lambda}=\frac{\partial^{2} \ell_{1}}{\partial \lambda \partial \alpha}=-k \sum_{i=1}^{m}\left(r_{i}+1\right) x_{(i)}^{\alpha} \log x_{(i)},
\end{aligned}
$$

and

$$
\frac{\partial^{2} \ell_{2}}{\partial p^{2}}=-\frac{\sum_{i=1}^{m-1} r_{i}}{p^{2}}-\frac{\left((m-1)(n-m)-\sum_{i=1}^{m-1}(m-i) r_{i}\right)}{(1-p)^{2}} .
$$

Since, the exact mathematical expressions for the elements of (13) is difficult to obtain. Thus, we give the approximate variance-covariance matrix for the MLEs, which is obtained by dropping the expectation operator $E$. Practically, we usually estimate $\mathrm{I}(\underline{\theta})$ by replaced $\underline{\theta}$ with $\underline{\hat{\theta}}$. Hence, the approximate variance-covariance matrix may be approximated as

$$
\mathrm{V}(\underline{\hat{\theta}}) \cong\left[\begin{array}{ccc}
\operatorname{Var}(\hat{\alpha}) & \operatorname{Cov}(\hat{\alpha}, \hat{\lambda}) & 0 \\
\operatorname{Cov}(\hat{\lambda}, \hat{\alpha}) & \operatorname{Var}(\hat{\lambda}) & 0 \\
0 & 0 & \operatorname{Var}(\hat{p})
\end{array}\right]
$$

where $\hat{\theta}=(\hat{\alpha}, \hat{\lambda}, \hat{p})^{\mathbf{T}}$. The asymptotic distribution of the MLEs $\underline{\hat{\theta}}$ is approximately with multivariate normal, i.e., $\underline{\hat{\theta}} \sim N(\underline{\theta}, \operatorname{Var}(\underline{\hat{\theta}}))$, see [18]. Then, $100(1-\gamma) \%$ two-sided ACIs for $\alpha, \lambda$ and $p$ are given, respectively, by

$$
\hat{\alpha} \pm z_{\gamma / 2} \sqrt{\operatorname{Var}(\hat{\alpha})}, \quad \hat{\lambda} \pm z_{\gamma / 2} \sqrt{\operatorname{Var}(\hat{\lambda})} \text { and } \hat{p} \pm z_{\gamma / 2} \sqrt{\operatorname{Var}(\hat{p})}
$$

where $\operatorname{Var}(\hat{\alpha}), \operatorname{Var}(\hat{\lambda})$ and $\operatorname{Var}(\hat{p})$ are the main diagonal elements of (14), respectively, and $z_{\gamma / 2}$ is the percentile of the standard normal distribution with right-tail probability $(\gamma / 2)-t h$.

\section{Bayes Procedure}

In this section, we have obtained the BEs of the unknown parameters $\alpha, \lambda$ and $p$ under progressively first-failure censored data with binomial removals. According to [17,23], we assume that $\alpha, \lambda$ and $p$ are random variables which are independently distributed having PDFs, respectively, as

$$
\begin{aligned}
& \pi_{1}(\alpha)=\frac{b_{1}^{a_{1}}}{\Gamma\left(a_{1}\right)} \alpha^{a_{1}-1} e^{-b_{1} \alpha}, \quad \alpha>0, a_{1}, b_{1}>0, \\
& \pi_{2}(\lambda)=\frac{b_{2}^{a_{2}}}{\Gamma\left(a_{2}\right)} \lambda^{a_{2}-1} e^{-b_{2} \lambda}, \quad \lambda>0, a_{2}, b_{2}>0,
\end{aligned}
$$




$$
\pi_{3}(p)=\frac{1}{B\left(a_{3}, b_{3}\right)} p^{a_{3}-1}(1-p)^{b_{3}-1}, \quad 0<p<1, a_{3}, b_{3}>0 .
$$

From (15), the joint PDF of $\alpha$ and $\lambda$ can be written with proportional as

$$
\pi(\alpha, \lambda) \propto \alpha^{a_{1}-1} \lambda^{b_{1}-1} e^{-\left(b_{1} \alpha+b_{2} \lambda\right)}, \quad \alpha, \lambda>0, a_{1}, b_{1}, a_{2}, b_{2}>0 .
$$

In continuous Bayes' theorem, the joint posterior PDF of $\alpha$ and $\lambda$ will be

$$
\pi^{*}(\alpha, \lambda \mid x, r)=\frac{L_{1}(\alpha, \lambda \mid x, r) \pi(\alpha, \lambda)}{\int_{0}^{\infty} \int_{0}^{\infty} L_{1}(\alpha, \lambda \mid x, r) \pi(\alpha, \lambda) d \alpha d \lambda} .
$$

Combining (9) with (17), the joint posterior distribution (18) can be written with proportional as

$$
\pi_{1}^{*}(\alpha, \lambda \mid x, r) \propto\left(A(\alpha) \alpha^{m+a_{1}-1} e^{-b_{1} \alpha}\right)\left(\lambda^{m+a_{2}-1} e^{-b_{2}^{*} \lambda}\right),
$$

where $A(\alpha)=\prod_{i=1}^{m} x_{(i)}^{\alpha}$ and $b_{2}^{*}=\left(b_{2}+k \sum_{i=1}^{m}\left(r_{i}+1\right) x_{(i)}^{\alpha}\right)$. The normalizing constant, $C_{1}^{*}$, of (19) is given by

$$
C_{1}^{*}=\int_{0}^{\infty} \int_{0}^{\infty}\left(A(\alpha) \alpha^{m+a_{1}-1} e^{-b_{1} \alpha}\right)\left(\lambda^{m+a_{2}-1} e^{-b_{2}^{*} \lambda}\right) d \alpha d \lambda .
$$

Similarly, combining (6) with (16), the posterior PDF of $p$ becomes

$$
\pi_{2}^{*}(p \mid \mathbf{R}=r)=\frac{1}{B\left(a_{3}^{*}, b_{3}^{*}\right)} p^{a_{3}^{*}-1}(1-p)^{b_{3}^{*}-1},
$$

where $a_{3}^{*}=a_{3}+\sum_{i=1}^{m-1} r_{i}$ and $b_{3}^{*}=b_{3}+(m-1)(n-m)-\sum_{i=1}^{m-1}(m-i) r_{i}$.

Hence, the marginal PDFs of $\alpha$ and $\lambda$, are given, respectively, by

$$
\begin{gathered}
f(\alpha \mid x, r)=\frac{A(\alpha) \alpha^{m+a_{1}-1} e^{-b_{1} \alpha}}{C_{2}^{*}\left(b_{2}^{*}\right)^{m+a_{2}}}, \quad \alpha>0, \\
f(\lambda \mid x, r)=\frac{\lambda^{m+a_{2}-1} e^{-b_{2}^{*} \lambda}}{C_{3}^{*}} \int_{0}^{\infty} A(\alpha) \alpha^{m+a_{1}-1} e^{-b_{1} \alpha} d \alpha, \quad \lambda>0,
\end{gathered}
$$

where $C_{2}^{*}$ and $C_{3}^{*}$ of (21), are given, respectively, by

$$
\begin{gathered}
C_{2}^{*}=\int_{0}^{\infty} \frac{A(\alpha) \alpha^{m+a_{1}-1} e^{-b_{1} \alpha}}{\left(b_{2}^{*}\right)^{m+a_{2}}} d \alpha, \\
C_{3}^{*}=\int_{0}^{\infty} \int_{0}^{\infty} A(\alpha) \alpha^{m+a_{1}-1} \lambda^{m+a_{2}-1} e^{-\left(b_{1} \alpha+b_{2}^{*} \lambda\right)} d \alpha d \lambda .
\end{gathered}
$$

A very well-known symmetric loss function is the SEL function, where $\tilde{\theta}_{s}$ being an estimate of $\theta$, is defined as $l_{s}(\theta, \tilde{\theta})=(\tilde{\theta}-\theta)^{2}$. Under this loss, the BE is the posterior mean of $\theta$. Based on (19), the BEs of the parameters $\alpha$ and $\lambda$ against SEL function cannot be computed analytically, because they do not result in closed forms. Therefore, we propose the use of numerical integration method by using MCMC techniques namely Gibbs sampler (see [22]), and M-H algorithm (see [13]) can be effectively used to generate a samples from the respective posterior distributions (19) and (20), and in turn to compute the BEs of $\alpha, \lambda$ and $p$ as well as construct the associated $100(1-\gamma) \%$ two-sided BCIs. From (19), the full conditional forms of $\alpha$ and $\lambda$ can be written, respectively, as

$$
\begin{gathered}
\pi(\alpha \mid x, \lambda) \propto A(\alpha) \alpha^{m+a_{1}-1} e^{-b_{1} \alpha}, \\
\lambda \mid x, \alpha \sim \operatorname{Gamma}\left(m+a_{2}, b_{2}^{*}\right) .
\end{gathered}
$$


From (22), the samples of $\lambda$ can be easily generated using any gamma generating routine. But, the full conditional distribution of $\alpha$, cannot be reduced analytically to well-known distributions. Therefore, the $\mathrm{M}-\mathrm{H}$ algorithm with normal proposal distribution will be used. The detailed procedure can be described as

Step 1: Start with initial guess $\alpha^{(0)}, \lambda^{(0)}$ and $p^{(0)}$.

Step 2: Set $J=1$.

Step 3: Generate $p^{(J)}$ from $\operatorname{Beta}\left(a_{3}^{*}, b_{3}^{*}\right)$.

Step 4: Generate $\lambda^{(J)}$ from Gamma $\left(m+a_{2}, b_{2}^{*}\right)$.

Step 5: Using M-H algorithm, generate $\alpha^{(J)}$ from $\pi\left(\alpha^{(J-1)} \mid x, \lambda^{(J)}\right)$ with the proposal distribution $q(\alpha) \equiv$ $N(\hat{\alpha}, \operatorname{Var}(\hat{\alpha}))$ as follows:

(a) Let $v=\alpha^{(J-1)}$, here we set $\alpha^{(0)} \equiv \hat{\alpha}$.

(b) Generate $\omega$ from the proposal distribution $q(\alpha)$.

(c) Let $\varphi(v, \omega)=\min \left\{1, \frac{\pi(\omega \mid x) q(v)}{\pi(v \mid x) q(\omega)}\right\}$.

(d) Accept $\omega$ with probability $\varphi(v, \omega)$ or accept $v$ with probability $1-\varphi(v, \omega)$.

Step 6: Set $J=J+1$.

Step 7: Repeat Steps 3-6 for $M$ times and obtain $\alpha^{(J)}, \lambda^{(J)}$ and $p^{(J)}$ for $J=1,2, \ldots, M$.

In order to guarantee the convergence, the first simulated varieties, $M_{0}$, of the algorithm may be biased by the initial value, therefore, usually discarded in the beginning of the analysis implementation (burn-in period). Then the selected samples are $\alpha^{(J)}, \lambda^{(J)}$ and $p^{(J)}$ for $J=M_{0}+1, \ldots, M$, are sufficiently large $M$. Then, the BEs of the parameter vector $\underline{\theta}$, is given by $\underline{\tilde{\theta}}_{s}=\sum_{J=M_{0}+1}^{M} \underline{\theta}^{(J)} /\left(M-M_{0}\right)$. To compute the BCIs of $\underline{\theta}$, order $\underline{\theta}^{(J)}$ as $\left(\underline{\theta}^{(1)}, \underline{\theta}^{(2)}, \ldots, \underline{\theta}^{(M)}\right)$, then $100(1-\gamma) \%$ two-sided BCIs of $\underline{\theta}$ is given by $\left(\underline{\theta}^{[M(\gamma / 2)]}, \underline{\theta}^{[M(1-(\gamma / 2))]}\right)$.

\section{A Simulation Study}

To study the effectiveness of the estimators obtained in previous sections, we need to simulate progressively firstfailure censored samples with binomial removals from specified WD. All necessary computational algorithms were coded in $\mathrm{R}$ statistical programming language software and were done on a laptop computer with a Core(TM) i5-2410M processor and 4.00 gigabytes of RAM. To implementing the computations, we used two useful packages are CODA package, which provides functions for summarizing and plotting the output of MCMC simulations, proposed by [21], and maxLik package, which using Newton-Raphson method of maximization in the computations, proposed by [14]. To get such a sample, use the following algorithm:

Step 1: Fix the values of $n, m, \alpha, \lambda$ and $p$.

Step 2: Generate a value from the prior densities of $\alpha, \lambda$ and $p$.

Step 3: Generate a random number $r_{1}$ from $\operatorname{Bin}(n-m, p)$.

Step 4: Generate random number $r_{i}$ from $\operatorname{Bin}\left(n-m-\sum_{j=1}^{i-1} r_{j}, p\right)$, for $i=2,3, \ldots, m-1$.

Step 5: Set $r_{m}$ according to the following relation:

$$
r_{m}= \begin{cases}n-m-\sum_{j=1}^{m-1} r_{j}, & \text { if } n-m-\sum_{j=1}^{m-1} r_{j}>0, \\ 0, & \text { for otherwise. }\end{cases}
$$

Step 6: Given $R=r$, generate a Type-II PCS from $U(0,1)$ by using the algorithm described in [6].

Step 7: For given values of $\alpha$ and $\lambda$, set $x_{(i)}=F^{-1}(U), i=1,2, \ldots, m$. Then, $x_{(i)}, i=1,2, \ldots, m$ is the required progressively first-failure censored sample with binomial removals of size $m$ from the WD.

For a different combinations of $n, m$ and $k$, we generate 1000 progressively first-failure-censored samples from the WD with arbitrarily true values $(\alpha, \lambda, p)=(1,1,0.5)$. we generate 1000 progressively first-failure-censored samples from the WD with arbitrarily true values $\alpha, \lambda$ and $p$ are estimated on the basis of Monte Carlo simulation study of 1000 samples. Different tests are considered with $n=20$ (small), $n=40$ (moderate) and $n=80$ (large), as 
well as different group sizes $k=1,3$ and 5 . The test is terminated when the number of failed subjects achieves or exceeds a certain value $m$, where the failure proportion $m / n=30,60$ and $90 \%$.

In Bayesian analysis, the choice of the hyper parameters is the main issue. The choice value of hyper parameters for the unknown parameters is made by assuming two independent information, namely prior mean and prior variance of $\alpha, \lambda$ and $p$. For computing the BEs, we used informative and non-informative priors for both the shape and scale parameters. If $a_{i}=b_{i}=0, i=1,2,3$, it is called prior (0), in this case the posterior distribution is proportional to the likelihood function. Other than prior (0), we used two different informative priors, are called, prior (1): $a_{i}=b_{i}=1, i=1,2,3$, and prior (2): $a_{i}=b_{i}=5, i=1,2,3$. Using the simulation algorithm proposed in Section 3, we generate 10,000 MCMC samples, and then the first 2000 iterations (burn-in period) have been discarded from the generated sequence. The average BEs under SEL function are computed based on 8,000 MCMC samples. The average values of the MLEs and BEs of $\alpha$ and $\lambda$ along with their MSEs are computed and summarized in Tables 2 and 3, respectively. Also, we report the ALs of 95\% ACIs/BCIs in Table 4. Since (6) and (20) does not depend on the group size $k$, then the average values of the MLEs and BEs of $p$ along with their MSEs are computed, for different choices of $n$ and $m$ and listed in Table 5. The associated ALs of 95\% ACIs/BCIs of $p$ are listed in Table 6.

Table 2. The average estimates of $\alpha$ with their MSEs (in parentheses).

\begin{tabular}{|c|c|c|c|c|c|c|}
\hline \multirow[t]{2}{*}{$k$} & \multirow[t]{2}{*}{$n$} & \multirow[t]{2}{*}{$m$} & \multirow[t]{2}{*}{ MLE } & \multicolumn{3}{|c|}{$\overline{\mathrm{BE}}$} \\
\hline & & & & Prior (0) & Prior (1) & Prior (2) \\
\hline \multirow[t]{9}{*}{1} & \multirow[t]{3}{*}{20} & 18 & $1.0934(0.0087)$ & $0.9204(0.0063)$ & $0.9488(0.0026)$ & $1.0435(0.0019)$ \\
\hline & & 12 & $1.1211(0.0147)$ & $0.9205(0.0063)$ & $1.0616(0.0038)$ & $1.0544(0.0029)$ \\
\hline & & 6 & $1.1259(0.0185)$ & $0.9166(0.0069)$ & $1.1009(0.0102)$ & $1.0814(0.0066)$ \\
\hline & \multirow[t]{3}{*}{40} & 36 & $1.0407(0.0017)$ & $0.9225(0.0055)$ & $1.0179(0.0003)$ & $1.0291(0.0008)$ \\
\hline & & 24 & $1.0423(0.0018)$ & $0.9324(0.0046)$ & $1.0309(0.0009)$ & $1.0378(0.0014)$ \\
\hline & & 12 & $1.0685(0.0047)$ & $0.9226(0.0059)$ & $1.0171(0.0003)$ & $1.0487(0.0023)$ \\
\hline & \multirow[t]{3}{*}{80} & 72 & $1.0198(0.0004)$ & $0.9300(0.0049)$ & $1.0083(0.0001)$ & $0.9666(0.0011)$ \\
\hline & & 48 & $1.0349(0.0012)$ & $0.9251(0.0056)$ & $1.0208(0.0004)$ & $1.0153(0.0002)$ \\
\hline & & 24 & $1.0355(0.0013)$ & $0.9264(0.0054)$ & $1.0349(0.0012)$ & $1.0103(0.0001)$ \\
\hline \multirow[t]{9}{*}{3} & \multirow[t]{3}{*}{20} & 18 & $1.0812(0.0066)$ & $0.8573(0.0204)$ & $1.0390(0.0015)$ & $1.0339(0.0012)$ \\
\hline & & 12 & $1.0271(0.0105)$ & $0.8559(0.0207)$ & $1.0625(0.0039)$ & $1.0545(0.0029)$ \\
\hline & & 6 & $1.1359(0.0185)$ & $0.8540(0.0213)$ & $1.0487(0.0024)$ & $1.0705(0.0049)$ \\
\hline & \multirow[t]{3}{*}{40} & 36 & $1.0374(0.0014)$ & $0.8684(0.0173)$ & $1.0313(0.0010)$ & $1.0268(0.0007)$ \\
\hline & & 24 & $1.0437(0.0019)$ & $0.8722(0.0163)$ & $1.0382(0.0014)$ & $1.0253(0.0006)$ \\
\hline & & 12 & $1.0608(0.0037)$ & $0.8706(0.0167)$ & $1.0385(0.0015)$ & $1.0327(0.0011)$ \\
\hline & \multirow[t]{3}{*}{80} & 72 & $1.0156(0.0003)$ & $0.8572(0.0204)$ & $1.0084(0.0001)$ & $0.9856(0.0002)$ \\
\hline & & 48 & $1.0315(0.0010)$ & $0.8655(0.0181)$ & $1.0407(0.0016)$ & $1.0382(0.0015)$ \\
\hline & & 24 & $1.0378(0.0014)$ & $0.8065(0.0375)$ & $0.9736(0.0007)$ & $1.0328(0.0011)$ \\
\hline \multirow[t]{9}{*}{5} & \multirow[t]{3}{*}{20} & 18 & $1.0767(0.0059)$ & $0.9056(0.0089)$ & $1.0973(0.0004)$ & $1.0409(0.0016)$ \\
\hline & & 12 & $1.1036(0.0107)$ & $0.9287(0.0051)$ & $1.0549(0.0030)$ & $1.0505(0.0026)$ \\
\hline & & 6 & $1.1359(0.0184)$ & $0.9644(0.0013)$ & $1.0973(0.0095)$ & $1.0615(0.0038)$ \\
\hline & \multirow[t]{3}{*}{40} & 36 & $1.0280(0.0008)$ & $0.8622(0.0189)$ & $0.9858(0.0002)$ & $1.0295(0.0009)$ \\
\hline & & 24 & $1.0387(0.0015)$ & $0.8582(0.0200)$ & $0.9839(0.0003)$ & $1.0352(0.0012)$ \\
\hline & & 12 & $1.0596(0.0035)$ & $0.8562(0.0207)$ & $1.0415(0.0017)$ & $0.9796(0.0004)$ \\
\hline & \multirow[t]{3}{*}{80} & 72 & $1.0156(0.0002)$ & $0.8473(0.0233)$ & $0.9711(0.0008)$ & $1.0198(0.0004)$ \\
\hline & & 48 & $1.0298(0.0009)$ & $0.8618(0.0191)$ & $1.0227(0.0005)$ & $0.9709(0.0008)$ \\
\hline & & 24 & $1.0353(0.0012)$ & $0.8738(0.0159)$ & $1.0304(0.0009)$ & $1.0201(0.0004)$ \\
\hline
\end{tabular}


Table 3. The average estimates of $\lambda$ with their MSEs (in parentheses).

\begin{tabular}{|c|c|c|c|c|c|c|}
\hline \multirow[t]{2}{*}{$k$} & \multirow[t]{2}{*}{$n$} & \multirow[t]{2}{*}{$m$} & \multirow[t]{2}{*}{ MLE } & \multicolumn{3}{|c|}{$\mathrm{BE}$} \\
\hline & & & & Prior (0) & Prior (1) & Prior (2) \\
\hline \multirow[t]{9}{*}{1} & 20 & 18 & $1.0416(0.0017)$ & $0.8799(0.0144)$ & $1.0407(0.0016)$ & $1.0332(0.0011)$ \\
\hline & & 12 & $1.0902(0.0081)$ & $0.8741(0.0158)$ & $1.0684(0.0047)$ & $1.0365(0.0013)$ \\
\hline & & 6 & $1.1674(0.0280)$ & $0.8726(0.0162)$ & $1.0661(0.0044)$ & $1.0317(0.0010)$ \\
\hline & 40 & 36 & $1.0125(0.0002)$ & $0.8794(0.0146)$ & $1.0108(0.0001)$ & $0.9742(0.0007)$ \\
\hline & & 24 & $1.0460(0.0021)$ & $0.8757(0.0155)$ & $0.9906(0.0001)$ & $1.0125(0.0002)$ \\
\hline & & 12 & $1.1241(0.0154)$ & $0.8727(0.0162)$ & $1.0931(0.0087)$ & $1.0165(0.0003)$ \\
\hline & 80 & 72 & $1.0093(0.0001)$ & $0.8775(0.0149)$ & $0.9859(0.0002)$ & $0.9894(0.0001)$ \\
\hline & & 48 & $1.0175(0.0003)$ & $0.8802(0.0143)$ & $1.0005(0.0001)$ & $1.0279(0.0007)$ \\
\hline & & 24 & $1.0375(0.0014)$ & $0.8735(0.0160)$ & $1.0354(0.0012)$ & $1.0206(0.0004)$ \\
\hline \multirow[t]{9}{*}{3} & 20 & 18 & $0.3395(0.4362)$ & $0.2313(0.5909)$ & $0.3736(0.3923)$ & $1.0314(0.0010)$ \\
\hline & & 12 & $0.3637(0.4049)$ & 0.2198 & $0.3561(0.4$ & 1.010 \\
\hline & & 6 & $0.3891(0.3732)$ & 0.2071 & $0.3532(0.4$ & 1.0209 \\
\hline & 40 & 36 & $0.3374(0.4390)$ & $0.2763(0.5237)$ & $0.3742(0.3916)$ & $0.9537(0.0021)$ \\
\hline & & 24 & $0.3471(0.4263)$ & $0.2745(0.5264)$ & $0.3734(0.3926)$ & $0.9825(0.0003)$ \\
\hline & & 12 & $0.3809(0.3833)$ & $0.2675(0.5365)$ & $0.3672(0.4004)$ & $0.9748(0.0006)$ \\
\hline & 80 & 72 & $0.3354(0.3354)$ & $0.2891(0.5054)$ & $0.3872(0.3756)$ & $0.8543(0.0212)$ \\
\hline & & 48 & $0.3383(0.4379)$ & $0.2828(0.5144)$ & $0.3764(0.3888)$ & $0.9612(0.0015)$ \\
\hline & & 24 & $0.3445(0.4297)$ & $0.2797(0.5188)$ & $0.3684(0.3989)$ & $1.0239(0.0006)$ \\
\hline \multirow[t]{9}{*}{5} & 20 & 18 & $0.2118(0.6213)$ & $0.2409(0.5762)$ & $0.3780(0.3868)$ & $0.9686(0.0009)$ \\
\hline & & 12 & $0.2105(0.6233)$ & $0.2313(0.5909)$ & $0.3716(0.3949)$ & $0.9603(0.0016)$ \\
\hline & & 6 & $0.2335(0.5876)$ & $0.2302(0.5926)$ & $0.3671(0.4006)$ & $1.0411(0.0017)$ \\
\hline & 40 & 36 & $0.2086(0.6262)$ & $0.2422(0.5742)$ & $0.3787(0.3859)$ & $0.8097(0.0362)$ \\
\hline & & 24 & $0.2087(0.6261)$ & $0.2357(0.5841)$ & $0.3757(0.3898)$ & $0.9397(0.0036)$ \\
\hline & & 12 & $0.2259(0.5993)$ & $0.2312(0.5909)$ & $0.3741(0.3917)$ & $1.0276(0.0008)$ \\
\hline & 80 & 72 & $0.2041(0.6333)$ & $0.2472(0.5667)$ & $0.3833(0.3804)$ & $0.5945(0.1644)$ \\
\hline & & 48 & $0.2040(0.6335)$ & $0.2424(0.5739)$ & $0.3689(0.3982)$ & $0.6555(0.1187)$ \\
\hline & & 24 & $0.2092(0.6254)$ & $0.2374(0.5815)$ & $0.3686(0.3991)$ & $0.9251(0.0056)$ \\
\hline
\end{tabular}

From the results presented in Tables 2-6, we have seen that the performance of the MLEs is quite close to that of the BEs under prior (0), as expected. Thus, if we have no prior information on the unknown parameters, then it is always better to use the MLEs rather than the BEs, because the BEs are computationally more expensive. In most cases, the BEs are very good than MLEs in terms of MSEs and ALs. If the effective sample size $m$ increases for given sample size $n$, the MSEs of the estimators of $\alpha, \lambda$ and $p$ decrease as failure proportion $m / n$ increases, as expect. Moreover, as the value of the group size $k$ increases, the MSEs associated with parameter $\alpha$ decrease while that associated with parameter $\lambda$ increase. Also, as $n$ increases, the ALs of 95\% ACIs/BCIs of $\alpha, \lambda$ and $p$ tend to decrease. It is also observed that the BCIs associate with $\alpha$ and $\lambda$ are better than ACIs in respect of ALs. Furthermore, the ALs of ACIs for $p$ are narrow down than BCIs. In general, it can be seen that, the BEs based on prior (2) has perform better than prior (1) in terms of minimum MSEs and ALs for all estimates. Because, the variance of prior (2) is smaller than prior (1), and both are more informative than the prior (0). Therefore, we recommend Bayesian point and interval estimation of the parameters using $\mathrm{M}-\mathrm{H}$ algorithm. 
Table 4. The ALs for $95 \%$ ACIs/BCIs of $\alpha$ and $\lambda$.

\begin{tabular}{|c|c|c|c|c|c|c|c|c|c|c|}
\hline \multirow[t]{3}{*}{$k$} & \multirow[t]{3}{*}{$n$} & \multirow[t]{3}{*}{$m$} & \multicolumn{4}{|c|}{$\alpha$} & \multicolumn{4}{|c|}{$\lambda$} \\
\hline & & & \multirow[t]{2}{*}{ ACI } & \multicolumn{3}{|c|}{ BCI } & \multirow[t]{2}{*}{ ACI } & \multicolumn{3}{|c|}{ BCI } \\
\hline & & & & $\begin{array}{l}\text { Prior } \\
(0)\end{array}$ & $\begin{array}{l}\text { Prior } \\
\text { (1) }\end{array}$ & $\begin{array}{l}\text { Prior } \\
\text { (2) }\end{array}$ & & $\begin{array}{l}\text { Prior } \\
(0)\end{array}$ & $\begin{array}{l}\text { Prior } \\
\text { (1) }\end{array}$ & $\begin{array}{l}\text { Prior } \\
(2)\end{array}$ \\
\hline \multirow[t]{9}{*}{1} & \multirow[t]{3}{*}{20} & 18 & 0.7398 & 0.2182 & 0.1074 & 0.0959 & 0.9673 & 0.1942 & 0.1248 & 0.0990 \\
\hline & & 12 & 0.8112 & 0.2208 & 0.1270 & 0.1077 & 1.2720 & 0.2096 & 0.1094 & 0.0952 \\
\hline & & 6 & 0.9025 & 0.2259 & 0.1417 & 0.1177 & 1.8861 & 0.2099 & 0.1144 & 0.0953 \\
\hline & \multirow[t]{3}{*}{40} & 36 & 0.5242 & 0.2124 & 0.1048 & 0.1007 & 0.9363 & 0.2127 & 0.0976 & 0.0854 \\
\hline & & 24 & 0.5339 & 0.2055 & 0.0849 & 0.1005 & 0.8724 & 0.2089 & 0.1095 & 0.0941 \\
\hline & & 12 & 0.7905 & 0.2075 & 0.1040 & 0.1016 & 1.2756 & 0.2198 & 0.0933 & 0.0931 \\
\hline & \multirow[t]{3}{*}{80} & 72 & 0.3707 & 0.2051 & 0.0971 & 0.0991 & 0.4881 & 0.2105 & 0.0984 & 0.0791 \\
\hline & & 48 & 0.4056 & 0.2107 & 0.0861 & 0.1063 & 0.6343 & 0.2073 & 0.0727 & 0.0661 \\
\hline & & 24 & 0.5272 & 0.2104 & 0.1013 & 0.0962 & 0.8309 & 0.2098 & 0.0534 & 0.0729 \\
\hline \multirow[t]{9}{*}{3} & \multirow[t]{3}{*}{20} & 18 & 0.7102 & 0.2148 & 0.0977 & 0.0851 & 0.3155 & 0.2072 & 0.0949 & 0.0949 \\
\hline & & 12 & 0.7594 & 0.2211 & 0.1249 & 0.0924 & 0.4134 & 0.2069 & 0.0897 & 0.0954 \\
\hline & & 6 & 0.8949 & 0.2205 & 0.1363 & 0.0999 & 0.6234 & 0.2142 & 0.0885 & 0.0964 \\
\hline & \multirow[t]{3}{*}{40} & 36 & 0.5039 & 0.2076 & 0.0943 & 0.0554 & 0.2217 & 0.2054 & 0.0898 & 0.0790 \\
\hline & & 24 & 0.5419 & 0.2063 & 0.0793 & 0.0949 & 0.2806 & 0.2071 & 0.0989 & 0.0762 \\
\hline & & 12 & 0.7915 & 0.2028 & 0.1006 & 0.0727 & 0.4317 & 0.2172 & 0.0948 & 0.0923 \\
\hline & \multirow[t]{3}{*}{80} & 72 & 0.3643 & 0.2056 & 0.0869 & 0.0776 & 0.1551 & 0.2059 & 0.0752 & 0.0705 \\
\hline & & 48 & 0.3776 & 0.2084 & 0.0791 & 0.0726 & 0.1924 & 0.2097 & 0.0757 & 0.0634 \\
\hline & & 24 & 0.5270 & 0.2099 & 0.0999 & 0.0889 & 0.2757 & 0.2043 & 0.0629 & 0.0872 \\
\hline \multirow[t]{9}{*}{5} & \multirow[t]{3}{*}{20} & 18 & 0.6867 & 0.2138 & 0.0834 & 0.0672 & 0.1958 & 0.2063 & 0.0945 & 0.0821 \\
\hline & & 12 & 0.7588 & 0.2120 & 0.1218 & 0.0822 & 0.2391 & 0.2010 & 0.0866 & 0.0875 \\
\hline & & 6 & 0.8943 & 0.2050 & 0.1369 & 0.1003 & 0.3737 & 0.2113 & 0.0804 & 0.0717 \\
\hline & \multirow[t]{3}{*}{40} & 36 & 0.3977 & 0.2109 & 0.0782 & 0.0576 & 0.1373 & 0.2046 & 0.0839 & 0.0846 \\
\hline & & 24 & 0.5304 & 0.2083 & 0.0731 & 0.0802 & 0.1672 & 0.2094 & 0.0959 & 0.0819 \\
\hline & & 12 & 0.7749 & 0.2066 & 0.0978 & 0.0924 & 0.2565 & 0.2105 & 0.0856 & 0.0934 \\
\hline & \multirow[t]{3}{*}{80} & 72 & 0.3553 & 0.2099 & 0.0749 & 0.0753 & 0.0945 & 0.2082 & 0.0635 & 0.0532 \\
\hline & & 48 & 0.3578 & 0.2081 & 0.0760 & 0.0864 & 0.1159 & 0.2066 & 0.0790 & 0.0565 \\
\hline & & 24 & 0.5236 & 0.2093 & 0.0794 & 0.0808 & 0.1675 & 0.2015 & 0.0825 & 0.0588 \\
\hline
\end{tabular}

\section{Real Data Analysis}

Here, we consider the real-life data set presented by [19] to illustrate the proposed methods in the previous sections. This data set consists of 30 failure times of the air conditioning system of an airplane. [12] analyzed this data set. Recently, [9, 3] fitted the WD for this real data set and concluded that the data are coming from the WD. To compute the MLEs, we plot the profile log-likelihood function in Figure 1 to obtain the initial guess of $\alpha$. Figure 1 shows that the profile log-likelihood function is unimodal, so we suggest using $\alpha=0.1$ as an initial value to start the iteration to obtain the MLEs. The ordered data of 30 failure times are: 1, 3, 5, 7, 11, 11, 11, 12, 14, 14, 14, $16,16,20,21,23,42,47,52,62,71,71,87,95,90,120,120,225,246,261$. Using this sample, a progressively first-failure censored sample is generated with binomial removals from the real data set with $m=10$ and $p=0.5$. Put this ordered data are in test, simultaneously, and randomly grouped into $n=15$ groups within $k=2$ items in each group. Using the algorithm discussed in Section 4, the generated sample from the real data set is presented in Table 7. The BEs of $\alpha, \lambda$ and $p$ can be obtained by run the chain for 6,000 iterations. Further, bun-in $M_{0}=1000$ samples to erase the effect of the initial values. 
Table 5. The average estimates of $p$ with their MSEs (in parentheses).

\begin{tabular}{cccccc}
\hline$n$ & $m$ & MLE & \multicolumn{3}{c}{ BE } \\
\cline { 4 - 6 } & & & Prior $(0)$ & Prior $(1)$ & Prior $(2)$ \\
\hline 20 & 18 & $0.0588(0.1946)$ & $0.0578(0.1969)$ & $0.0854(0.1739)$ & $0.1561(0.1213)$ \\
& 12 & $0.0908(0.1674)$ & $0.0920(0.1673)$ & $0.1001(0.1608)$ & $0.1342(0.1350)$ \\
& 6 & $0.1857(0.0988)$ & $0.1980(0.0934)$ & $0.2079(0.0876)$ & $0.2371(0.0714)$ \\
40 & 36 & $0.0286(0.2222)$ & $0.0275(0.2234)$ & $0.0352(0.2163)$ & $0.0587(0.1951)$ \\
& 24 & $0.0435(0.2084)$ & $0.0437(0.2082)$ & $0.0465(0.2058)$ & $0.0565(0.1968)$ \\
& 12 & $0.0909(0.1674)$ & $0.0913(0.1673)$ & $0.0941(0.1650)$ & $0.1035(0.1575)$ \\
80 & 72 & $0.0141(0.2361)$ & $0.0144(0.2358)$ & $0.0159(0.2344)$ & $0.0229(0.2277)$ \\
& 48 & $0.0213(0.2292)$ & $0.0214(0.2291)$ & $0.0221(0.2284)$ & $0.0246(0.2261)$ \\
& 24 & $0.0435(0.2084)$ & $0.0436(0.2083)$ & $0.0444(0.2076)$ & $0.0469(0.2053)$ \\
\hline
\end{tabular}

Table 6. The ALs for $95 \%$ ACIs/BCIs of $p$.

\begin{tabular}{cccccc}
\hline$n$ & $m$ & ACI & \multicolumn{3}{c}{ BCI } \\
\cline { 4 - 6 } & & & Prior (0) & Prior (1) & Prior (2) \\
\hline 20 & 18 & 0.1381 & 0.1383 & 0.1711 & 0.2096 \\
& 12 & 0.1206 & 0.1204 & 0.1207 & 0.1339 \\
& 6 & 0.1854 & 0.1812 & 0.1885 & 0.1913 \\
40 & 36 & 0.0552 & 0.0531 & 0.0609 & 0.0754 \\
& 24 & 0.0417 & 0.0415 & 0.0421 & 0.0462 \\
& 12 & 0.0645 & 0.0635 & 0.0665 & 0.0684 \\
80 & 72 & 0.0194 & 0.0189 & 0.0199 & 0.0239 \\
& 48 & 0.0146 & 0.0140 & 0.0149 & 0.0158 \\
& 24 & 0.0223 & 0.0218 & 0.0224 & 0.0232 \\
\hline
\end{tabular}

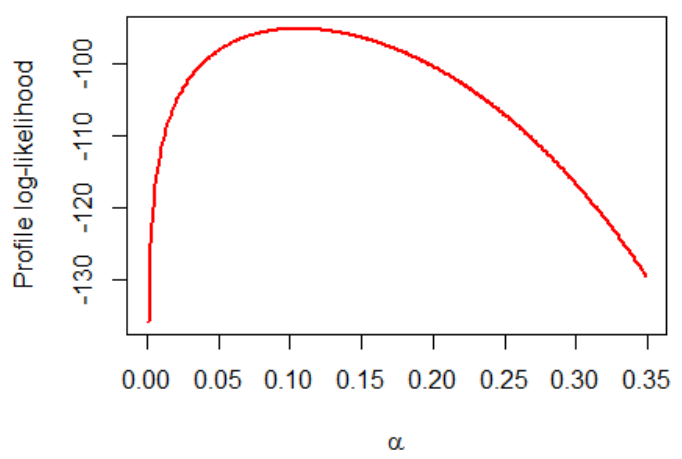

Figure 1. Profile log-likelihood function of $\alpha$

Moreover, some important characteristics such as: mean, median, mode, standard deviation (SD), standard error (SE) and skewness (Sk.) for MCMC posterior distributions of $\alpha, \lambda$ and $p$ after bun-in; are computed and provided in Table 8. Since, we have no prior information about the unknown parameters, we assume that the non-informative priors of the unknown parameters. The MLEs, BEs as well as 95\% ACIs/BCIs of $\alpha, \lambda$ and $p$ are computed and reported in Table 9. Also, the trace plots of MCMC outputs for posterior distribution of $\alpha, \lambda$ and $p$ for the real data set are plotted in Figure 2, which indicates that the MCMC procedure converges very well. Table 8 shows that the 
SEs of the Bayes MCMC estimates are very close to zero. Furthermore, the histograms and the posterior density functions for the MCMC output of $\alpha, \lambda$ and $p$ are plotted in Figure 3. It is observed that the histograms of the generated posteriors match quite well with the theoretical posterior density functions.
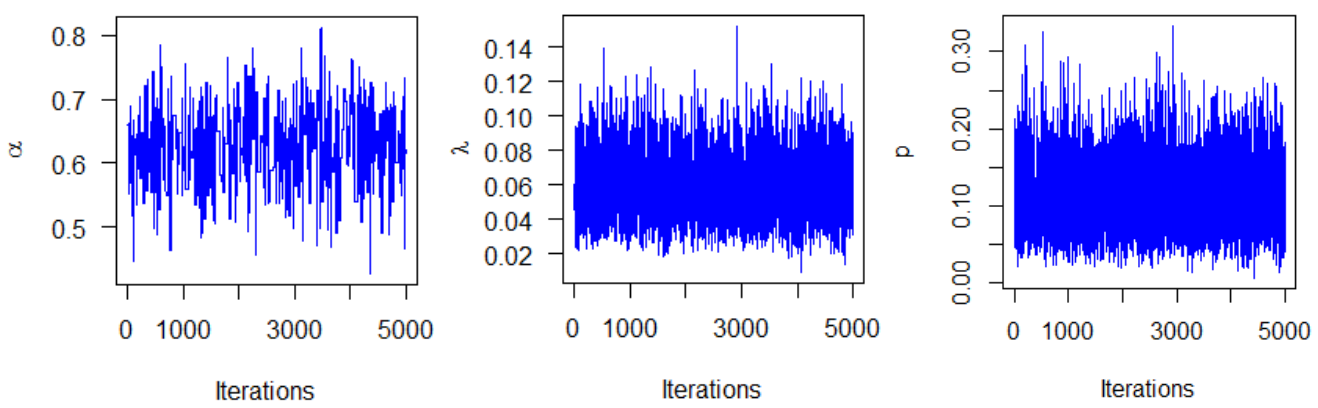

Figure 2. Trace plots of $\alpha, \lambda$ and $p$ obtained from MCMC method.


Figure 3. Histogram of $\alpha, \lambda$ and $p$ generated by MCMC method.

Table 7. A generated progressively first-failure censored sample with binomial removals.

\begin{tabular}{ccccccccccc}
\hline$i$ & 1 & 2 & 3 & 4 & 5 & 6 & 7 & 8 & 9 & 10 \\
\hline$r_{i}$ & 3 & 1 & 0 & 0 & 0 & 0 & 1 & 0 & 0 & 0 \\
\hline$x_{(i)}$ & 1 & 14 & 16 & 21 & 42 & 52 & 71 & 90 & 120 & 246 \\
\hline
\end{tabular}

Table 8. The MCMC results for some posterior characteristics.

\begin{tabular}{ccccccr}
\hline Parameter & Mean & Median & Mode & SD & SE & \multicolumn{1}{c}{ Sk. } \\
\hline$\alpha$ & 0.6244 & 0.6272 & 0.5894 & 0.060997 & 0.000863 & -0.090209 \\
$\lambda$ & 0.0574 & 0.0553 & 0.0089 & 0.018176 & 0.000257 & 0.649514 \\
$p$ & 0.1114 & 0.1055 & 0.0099 & 0.046898 & 0.000663 & 0.742705 \\
\hline
\end{tabular}


Table 9. The MLEs and BEs with their 95\% ACIs/BCIs.

\begin{tabular}{ccccc}
\hline Parameter & MLE & BE & ACI & BCI \\
\hline$\alpha$ & 0.9679 & 0.6244 & $(0.5108,1.4249)$ & $(0.5086,0.7323)$ \\
$\lambda$ & 0.0076 & 0.0574 & $(0.0000,0.0241)$ & $(0.0278,0.0977)$ \\
$p$ & 0.4167 & 0.1114 & $(0.1377,0.6956)$ & $(0.0378,0.2157)$ \\
\hline
\end{tabular}

\section{Concluding Remarks}

The purpose of this paper is to develop the estimation problems for the Weibull parameters when data are collected under PFFCS-BR, i.e., we extend the results of [26] in the case of WD under pre-fixed removals to the random removals, where the number of groups removed at each first-failure time follows a binomial distribution. Moreover, we generalized many several works and may be obtained as a special cases from the new results such as: [24] if putting $k=1$, [25] results in the case of exponential distribution by putting $\alpha=1$ and replacing $\lambda$ by $\theta^{-1}$, [10] results in the case of Rayleigh distribution by putting $\alpha=2$. Both the classical and Bayesian inference procedures for the parameters of WD have been discussed. Independent gamma priors are assumed for both the unknown two parameters of WD and provide the BEs relative to the SEL function. Since the MLEs and BEs cannot be obtained in closed forms, so the M-H algorithm and Gibbs sampler are considered. Also, 95\% two-sided ACIs/BCIs of the parameters $\alpha, \lambda$ and $p$ are constructed. A Monte Carlo simulation study was conducted to assess the performance of the MLEs and BEs. Using MCMC techniques with different values of $k, n$ and $m$, simulation results provided that the BEs based on non-informative prior are very similar the MLEs, but in the case of informative priors. In terms of minimum MSEs and ALs, the Bayesian procedure provides better estimates than MLEs. Also, we have seen that the MSEs of different BEs and MLEs of the unknown parameters are decreasing when the sample size $n$ and effective failure proportion $n / m$ increasing, as expect.

\section{Acknowledgement}

The authors would like to express thanks to the Associate Editor, Dr. Filipe J. Marques, and two anonymous referees for their constructive comments and suggestions, which significantly improved the paper. The authors would also like to thank the Coordinating Editor, Prof. David G. Yu for all his effort handling this paper.

\section{REFERENCES}

1. M. V. Ahmadi, and M. Doostparast, Pareto analysis for the lifetime performance index of products on the basis of progressively first-failure-censored batches under balanced symmetric and asymmetric loss functions, Journal of Applied Statistics, vol. 46, no. 7, pp. 1196-1227, 2019.

2. E. A. Ahmed, Estimation and prediction for the generalized inverted exponential distribution based on progressively first-failurecensored data with application, Journal of Applied Statistics, vol. 44, no. 9, pp. 1576-1608, 2017.

3. S. K. Ashour, and A. Elshahhat, Bayesian and non-Bayesian estimation for Weibull parameters based on generalized Type-II progressive hybrid censoring scheme, Pakistan Journal of Statistics and Operation Research, vol. 12, no. 2, pp. 213-226, 2016.

4. S. K. Ashour, A. A. El-Sheikh, and A. Elshahhat, Maximum likelihood estimation of the generalised Gompertz distribution under progressively first-failure censored sampling, South African Statistical Journal, vol. 52, no. 2, pp. 115-128, 2018.

5. N. Balakrishnan, and E. Cramer, The Art of Progressive Censoring. Applications to Reliability and Quality, New York, NY: Birkhäuser, 2014.

6. N. Balakrishnan, and R. A. Sandhu, A simple simulational algorithm for generating progressive Type-II censored samples, The American Statistician, vol. 49, no. 2, pp. 229-230, 1995.

7. U. Balasooriya, Failure-censored reliability sampling plans for the exponential distribution, Journal of Statistical Computation and Simulation, vol. 52, no. 4, pp. 337-349, 1995.

8. M. Chacko, and R. Mohan, Bayesian analysis of Weibull distribution based on progressive Type-II censored competing risks data with binomial removals, Computational Statistics, vol. 34, no. 1, pp. 233-252, 2019.

9. Y. Cho, and H. Sun, and K. Lee, Estimating the entropy of a Weibull distribution under generalized progressive hybrid censoring, Entropy, vol. 17, no. 1, pp. 102-122, 2015. 
10. S. Dey, and T. Dey, Statistical inference for the Rayleigh distribution under progressively Type-II censoring with binomial removal, Applied Mathematical Modelling, vol. 38, no. 3, pp. 974-982, 2014.

11. M. Dube, H. Krishna, and R. Garg, Generalized inverted exponential distribution under progressive first-failure censoring, Journal of Statistical Computation and Simulation, vol. 86, no. 6, pp. 1095-1114, 2016.

12. R. D. Gupta, and D. Kundu, Exponentiated exponential family: an alternative to gamma and Weibull distributions, Biometrical Journal: Journal of Mathematical Methods in Biosciences, vol. 43, no. 1, pp. 117-130, 2001.

13. W. K. Hastings, Monte Carlo sampling methods using Markov chains and their applications, Biometrika, vol. 57, no. 1, pp. 97-109, 1970.

14. A. Henningsen, and O. Toomet, maxLik: A package for maximum likelihood estimation in R, Computational Statistics, vol. 26, no. 3, pp. 443-458, 2011.

15. S.-R. Huang, and S.-J. Wu, Estimation of Pareto distribution under progressive first-failure censoring with random removals, Chinese Journal of Statistics, vol. 49, no. 3, pp. 82-97, 2011.

16. N. L. Johnson, S. Kotz, and N. Balakrishnan, Continuous Univariate Distributions, Wiley Series in Probability and Statistics, 1994.

17. A. Kaushik, U. Singh, and S. K. Singh, Bayesian inference for the parameters of Weibull distribution under progressive Type-I interval censored data with beta-binomial removals, Communications in Statistics-Simulation and Computation, vol. 46, no. 4, pp. 3140-3158, 2017.

18. J. F. Lawless, Statistical Models and Methods for Lifetime Data, John Wiley \& Sons, 2011.

19. H. Linhart, and W. Zucchini, Model Selection, John Wiley \& Sons, 1986.

20. H. S. Mohammed, S. F. Ateya, and E. K. AL-Hussaini, Estimation based on progressive first-failure censoring from exponentiated exponential distribution, Journal of Applied Statistics, vol. 44, no. 8, pp. 1479-1494, 2017.

21. M. Plummer, N. Best, K. Cowles, and K. Vines, CODA: convergence diagnosis and output analysis for MCMC, R news, vol. 6, no. 1 , pp. 7-11, 2006.

22. A. F. M. Smith, and G. O. Roberts, Bayesian computation via the Gibbs sampler and related Markov chain Monte Carlo methods, Journal of the Royal Statistical Society. Series B (Methodological), vol. 55, no. 1, pp. 3-23, 1993.

23. A. A. Soliman, A. H. Abd Ellah, N. A. Abou-Elheggag, and R. M. El-Sagheer, Estimation based on progressive first-failure censored sampling with binomial removals, Intelligent Information Management, vol. 5, no. 4, pp. 117-125, 2013.

24. S. K. Tse, C. Yang, and H.-K. Yuen, Statistical analysis of Weibull distributed lifetime data under Type-II progressive censoring with binomial removals, Journal of Applied Statistics, vol. 27, no. 8, pp. 1033-1043, 2000.

25. S.-J. Wu, and C.-T. Chang, Parameter estimations based on exponential progressive Type-II censored data with binomial removals, International Journal of Information and Management Sciences, vol. 13, no. 3, pp. 37-46, 2002.

26. S.-J. $\mathrm{Wu}$, and $\mathrm{C}$. Kus, On estimation based on progressive first-failure-censored sampling, Computational Statistics and Data Analysis, vol. 53, no. 10, pp. 3659-3670, 2009. 